\title{
Wall structure and energy on DWDD
}

\author{
A. Ohshima, K. Kusano, T. Kobayashi, Y. Fujiwara, S. Shiomi, and M. Kaneko* \\ Faculty of Engineering, Mie Univ., 1515 Kamihama-cho, Tsu, Mie, 514-8507, Japan \\ "Home Electronics Development Group, Sony Corp., 6-7-35 Kitashinagawa, Shinagawa-ku, Tokyo, 141-0001, Japan
}

Abstract- Wall structure and energy have been simulated for the Domain Wall Displacement Detection (DWDD), and the front process and the rear process under temperature gradient have been considered. For the front process, when the mark length is comparable with the domain wall width in the displacement (D) layer, the domain copied on the D-layer from the memory layer collapses. By making the D-layer thin, the minimum mark length for which the domain wall displacement occurs can be shortened. For the rear process, we confirmed that by inserting the control layer between the D-layer and the switching layer, the mark length with which the ghost signal is suppressed becomes longer. By making the anisotropy energy constant in the D-layer increase, improvement in the DWDD process is expected.

Key words: Domain Wall Displacement Detection, wall structure, wall energy, front process, rear process, domain wall displacement, ghost signal

\section{Introduction}

The Domain Wall Displacement Detection (DWDD) ${ }^{1), 2)}$ has been proposed as the readout method which can reproduce the minute mark beyond the resolution of the optical system.

The medium of the DWDD is composed of the displacement (denoted as D) layer, the switching (S) layer (the control (C) layer) and the memory (M) layer. The DWDD has two processes which consist of the front process and the rear process. The wall structure copied on the D-layer and the wall motion has been simulated for the $\mathrm{DWDD}^{3), 4)}$. In this paper, the front process and the rear process have been discussed from the viewpoint of the wall structure and the energy on the DWDD.

\section{Calculation method}

The layer structure of the DWDD with the Curie temperature $T_{c}$ of each layer is summarized in Fig. 1. Here, the C-layer is inserted between the D-layer and the S-layer in order to suppress the ghost signal on the rear process. The D-layer is made of amorphous Gd-TM alloy film, and other layers are made of amorphous Tb-TM alloy films.

The calculation model of the wall structure on the DWDD is the 2-dimensional model with the cell ( $\Delta x=\Delta z=$ $2.5 \mathrm{~nm}$ per side) arranged in the directions of the track and the film thickness. The energy $E$ per unit length stored in one cell is a function of the angle $(\theta, \phi)$ where $\theta$ is zenith and $\phi$ is azimuth as shown in Fig. 2. $E$ is calculated by

$$
\begin{aligned}
& E=\Delta x \Delta z\left(K_{\mathrm{u} i j}-2 \pi M_{\mathrm{sij}}{ }^{2}\right) \sin ^{2} \theta \\
& +2 \frac{A_{i j}+A_{i j-1}}{2}\left(1-\mathbf{m}_{i j} \cdot \mathbf{m}_{i j-1}\right)+2 \frac{A_{i j}+A_{i j+1}}{2}\left(1-\mathbf{m}_{i j} \cdot \mathbf{m}_{i j+1}\right) \\
& +2 \frac{A_{i j}+A_{i-1 j}}{2}\left(1-\mathbf{m}_{i j} \cdot \mathbf{m}_{i-1 j}\right)+2 \frac{A_{i j}+A_{i+1 j}}{2}\left(1-\mathbf{m}_{i j} \cdot \mathbf{m}_{i+1 j}\right)
\end{aligned}
$$

where $\mathbf{m}$ is the unit vector with the angle $(\theta, \phi), K_{\mathrm{u}}$ is the anisotropy energy constant, $A$ is the exchange stiffness constant, and subscript character $i j$ is the address of calculated cell. Excepting the M-layer, $(\theta, \phi)$ were determined by $\partial E / \partial \theta=0$ and $\partial E / \partial \phi=0$. In the M-layer, we set the two domain walls with a certain distance and fixed $(\theta, \phi)$ given by the analysis formula during the calculation for other layers. The directions of magnetic moment in the center of the two domain walls are considered to be in parallel or in anti-parallel. In this paper, we show the parallel case only, since the results of two cases have been almost the same.

Temperature profile is shown in Fig. 3, where linear velocity of medium is $3 \mathrm{~m} / \mathrm{sec}$. Plus and minus abscissas mean the front side and the rear side, respectively. Position $x=0$ indicates the center of light beam spot. Temperature gradient of the front side is larger than that of the rear side owing to the medium motion. Operations of the front side and the rear side are called as the front process and the rear process, respectively.

Temperature dependence of magnetic properties of each single layer was calculated by the ordinary mean field method $^{5)}$. The calculated magnetic properties of the magnetization, the domain wall energy, and the domain wall width of each single layer are shown in Fig. 4(a), Fig. 4(b), and Fig. 4(c), respectively.

\begin{tabular}{|ll|}
\hline Displacement layer & $T_{\mathrm{C}}=533 \mathrm{~K}$ \\
\hline (Control layer & $\left.T_{\mathrm{C}}=433 \mathrm{~K}\right)$ \\
\hline Switching layer & $T_{\mathrm{C}}=423 \mathrm{~K}$ \\
\hline Memory layer & $T_{\mathrm{C}}=588 \mathrm{~K}$ \\
\hline
\end{tabular}

Fig.1 Layer structure.

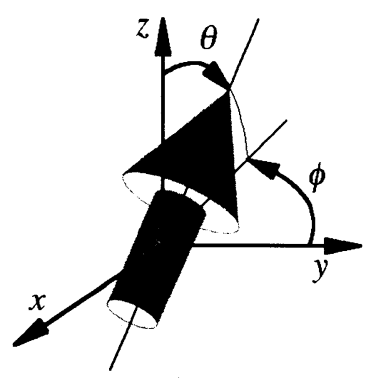

Fig.2 Coordinate.

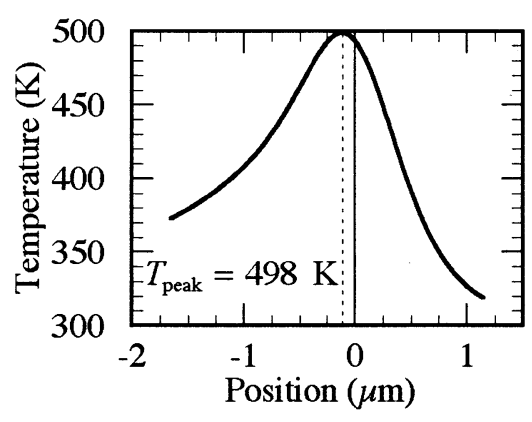

Fig.3 Temperature profile. 

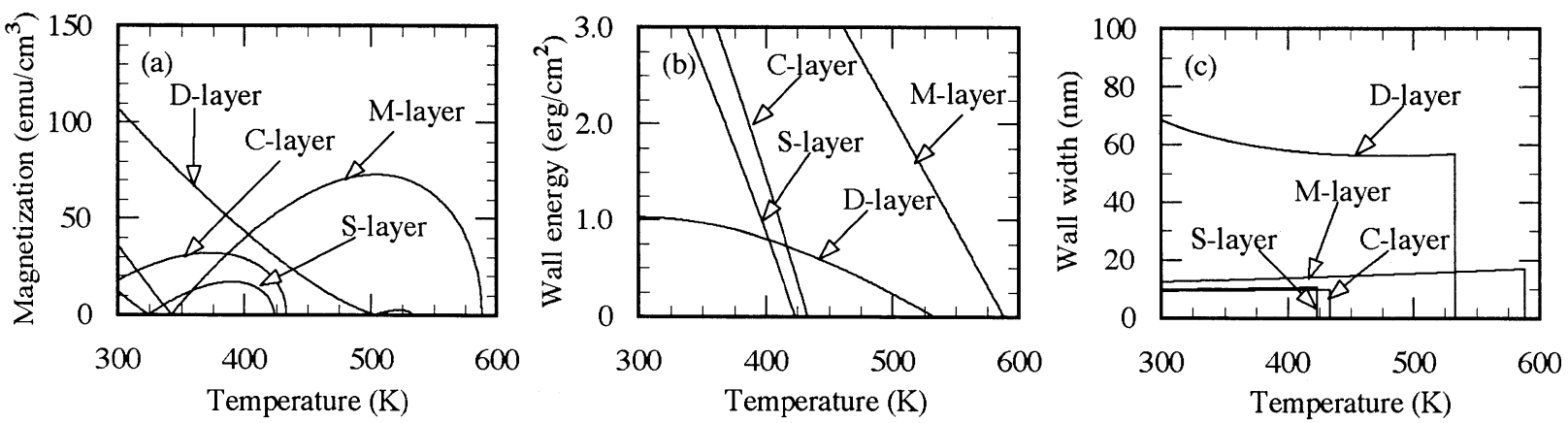

Fig.4 Temperature dependence of (a) Magnetizations, (b) domain wall energies, and (c) domain wall widths of each single layer.

\section{Calculation results}

\subsection{Wall structure at $300 \mathrm{~K}$}

The calculation result of wall structure copied from the M-layer at $300 \mathrm{~K}$ is shown in Fig. 5. Layer structure is the $\mathrm{D} / \mathrm{S} / \mathrm{M}$ structure for which the thicknesses of the D-layer and the S-layer are $30 \mathrm{~nm}$ and $10 \mathrm{~nm}$, respectively. The wall structure is represented by contour line and by gradation of the angle $\theta$ of the magnetic moment, where the dark areas correspond to wall. As shown in Fig. 4(c), the wall widths of the single D-layer and the single S-layer are about $70 \mathrm{~nm}$ and $10 \mathrm{~nm}$, respectively. When these layers are exchange-coupled, the wall width of the D-layer is narrowed at the interface due to high anisotropy of the S-layer.

\subsection{The front process}

The calculation results of wall structure in the front process are shown in Fig. 6, where the distance $d$ of the two domain walls in the M-layer, that is to say, mark length is (a) $70 \mathrm{~nm}$, or (b) $60 \mathrm{~nm}$. Layer structure is the same as Fig. 5. The left side of Fig. 6 is high temperature region. The temperature of hatched region is higher than the Curie temperature $T_{\mathrm{S}}$ of the S-layer, and three layers are not exchange-coupled each other on this hatched region. In the case of (a), when the non-coupling region approached the left wall, the left wall in the D-layer started to displace by temperature gradient, and reached the highest temperature position. However, in the case of (b), the two walls pulled each other, and finally, the domain copied on the D-layer collapsed. In other words, the two domain walls in the D-layer were transformed into the interface wall in the S-layer.

We calculated minimum mark length $d_{\min }$ for which the domain wall displacement can occur, and results are shown in Fig. 7, where calculation parameters are (a) the thickness $t_{\mathrm{D}}$ of the D-layer or $t_{\mathrm{S}}$ of the S-layer and (b) the anisotropy energy constant $K_{\mathrm{D}}$ of the D-layer. As a result, by making

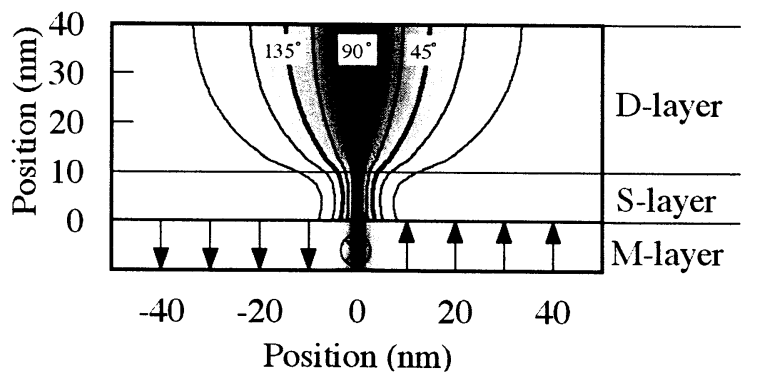

Fig.5 Wall structure at $300 \mathrm{~K}$.
$t_{\mathrm{D}}$ thin, $d_{\min }$ becomes shorter. However, dependence of $d_{\text {min }}$ on $t_{\mathrm{S}}$ can scarcely be seen. On the other hand, $d_{\text {min }}$ becomes shorter with increasing $K_{\mathrm{D}}$.

First of all, we consider magnetostatic energy. If domain is not surrounded by wall, and front and rear walls of domain are closely placed, a repulsion acts on the wall from the viewpoint of magnetostatic energy. This magnetostatic energy is not considered on this simulation. In the case of the DWDD, however, the repulsion may be neglected since the magnetization of the D-layer is set small.

Secondly, we evolved $d_{\min }$ by considering domain wall energy $\sigma_{\mathrm{d}}$ of the D-layer, interface wall energy $\sigma_{\mathrm{w}}$ created in the S-layer and coercivity energy $M_{\mathrm{D}} H_{\mathrm{D}}$ where $M_{\mathrm{D}}$ and $H_{\mathrm{D}}$ are the magnetization and the coercivity of the D-layer. When two domain walls stay in the D-layer, the total energy is approximated to $2 \sigma_{\mathrm{d}} t_{\mathrm{D}}$. On the other hand, when the walls are transformed into the interface wall, the total energy is $\sigma_{\mathrm{w}} d$. If the difference of energy $\Delta E_{\mathrm{tot}}$ between $2 \sigma_{\mathrm{d}} t_{\mathrm{D}}$ and $\sigma_{\mathrm{w}} d$ overcomes the energy barrier $2 M_{\mathrm{D}} H_{\mathrm{D}} t_{\mathrm{D}} d$ which needs to reverse magnetization and is attributed to $K_{\mathrm{D}}$, domain walls collapse and are transformed into the interface wall as shown in Fig. 6(b). That means $2 \sigma_{\mathrm{d}} t_{\mathrm{D}}-\sigma_{\mathrm{w}} d>2 M_{\mathrm{D}} H_{\mathrm{D}} t_{\mathrm{D}} d$. From this relationship, $d_{\min }$ is given by

$$
d_{\min }=\frac{2 \sigma_{\mathrm{d}} t_{\mathrm{D}}}{\sigma_{\mathrm{w}}+2 M_{\mathrm{D}} H_{\mathrm{D}} t_{\mathrm{D}}} .
$$

This $\Delta E_{\text {tot }}$ that corresponds to the coercivity encrgy $2 M_{\mathrm{D}} H_{\mathrm{D}} t_{\mathrm{D}} d_{\min }$ is shown in later discussion. Since $M_{\mathrm{D}}=$
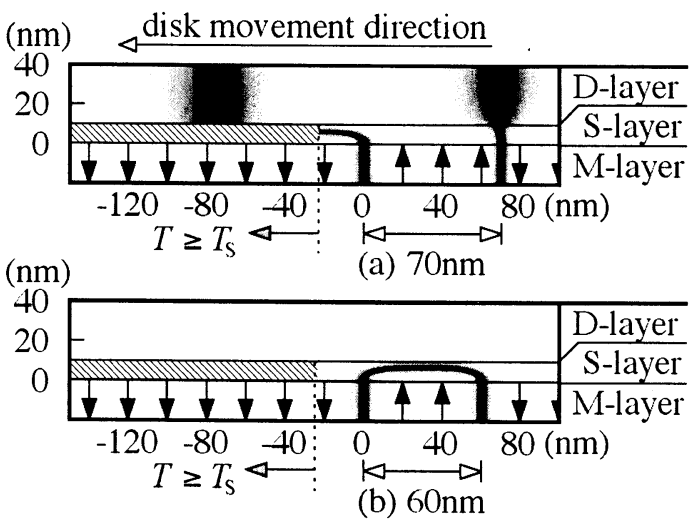

Fig.6 Wall structure in the front process. The distance $d$ of two Bloch walls in the M-layer is (a) $70 \mathrm{~nm}$, or (b) $60 \mathrm{~nm}$. 

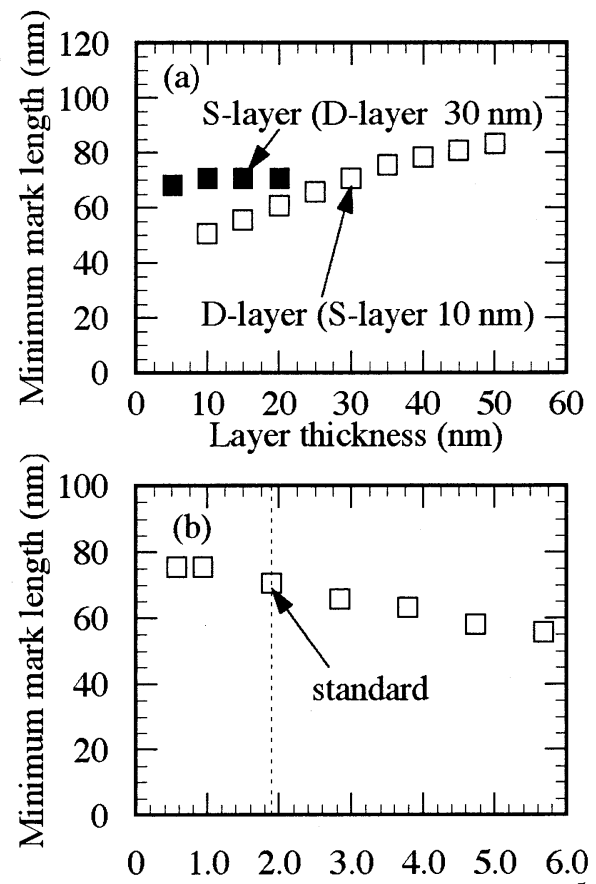

Anisotropy energy constant at $300 \mathrm{~K}\left(10^{5} \mathrm{erg} / \mathrm{cm}^{3}\right)$

Fig.7 Dependence of minimum mark length for which the domain wall displacement occurs in the front process on (a) layer thickness of the D-layer and the S-layer,

(b) anisotropy energy constant of the D-layer at $300 \mathrm{~K}$.

$40 \mathrm{emu} / \mathrm{cm}^{3}$ and $H_{\mathrm{D}}$ is estimated as $968 \mathrm{Oe}$ (see Eq. (3)), $H_{\mathrm{D}}$ is much larger than wall coercivity and much smaller than anisotropy field $H_{\mathrm{K}} \approx 2 K_{\mathrm{D}} / M_{\mathrm{D}}=5300$ Oe nevertheless the origin of $H_{\mathrm{D}}$ is $K_{\mathrm{D}}$.

Thinning $t_{\mathrm{D}}$, there is a tendency for $d_{\text {min }}$ to decrease from Eq. (2) since $\sigma_{\mathrm{w}} \neq 0$ at the time of wall displacement or domain collapse. This result is shown in Fig. 7(a). Also, from the relationship of $M_{\mathrm{D}} H_{\mathrm{D}} \propto K_{\mathrm{D}}$ and $\sigma_{\mathrm{d}} \approx 4 \sqrt{A_{\mathrm{D}} K_{\mathrm{D}}}$, if $\sigma_{\mathrm{w}}$ is suitably low, $d_{\min }$ becomes shorter by increasing $K_{\mathrm{D}}$. That agrees with the tendency of the calculated result shown in Fig. 7(b).

Finally, we treat an attraction that does not appear in the above discussions, and discuss the effect of the wall width. This attraction is effective when tails of walls overlap each other, and the attraction acts on wall due to decreasing wall energy that consists of anisotropy energy and exchange energy. Furthermore when tails of walls overlap, magnetic moment rotates from the easy direction. This means decrease of the coercivity energy $M_{\mathrm{D}} H_{\mathrm{D}}$, that is to say, $M_{\mathrm{D}} H_{\mathrm{D}}$ is a function of mark length $d$. Figure 8 shows total energy $E_{\text {tot }}$ of all cells in the D-layer and the S-layer as a function of mark length $d$ at $300 \mathrm{~K}$. This total energy $E_{\text {tot }}$ begins to decrease when the mark length is almost the same as the wall width. Therefore the attraction $\partial E_{\text {tot }} / \partial d$ begins to act when $d$ becomes shorter than the wall width of the D-layer. When layer structure is the same as Fig. 5 and the thickness of the D-layer is $30 \mathrm{~nm}$, for $d>60 \mathrm{~nm}$, $E_{\text {tot }}$ is twice as much as single wall energy. For $d<60$ $\mathrm{nm}, E_{\mathrm{tot}}$ decreases with decreasing $d$, and $\partial E_{\mathrm{tot}} / \partial d$ acts on the wall and increases with decreasing $d$ as shown in Fig. 8(a). Making $t_{\mathrm{D}}$ thin from $30 \mathrm{~nm}$ to $20 \mathrm{~nm}, \partial E_{\text {tot }} / \partial d$

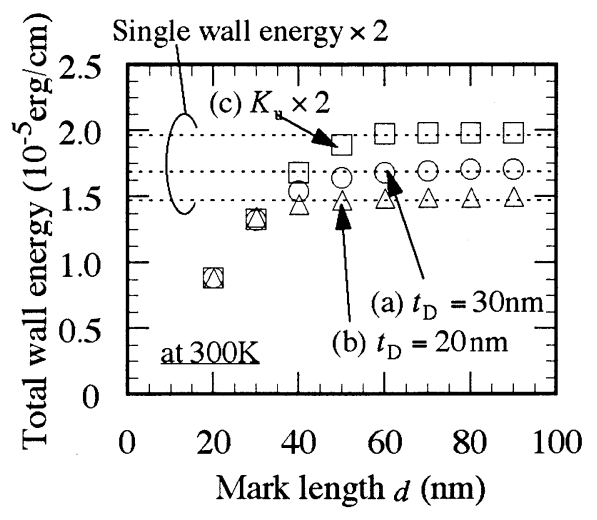

Fig.8 Total energy $E_{\text {tot }}$ of all cells in the D-layer and the S-layer as a function of mark length $d$ at $300 \mathrm{~K}$.

becomes weaker as shown in Fig. 8(b). This result is explained as follows. The wall width $\delta_{\mathrm{D}}$ of the D-layer is narrowed at the interface between the D-layer and the $\mathrm{S}$ layer, and broaden at the surface as shown in Fig. 5. If $t_{\mathrm{D}}$ is sufficiently thick, $\delta_{\mathrm{D}}$ at the surface becomes the intrinsic width of the D-layer determined by $\delta_{\mathrm{D}} \approx \pi \sqrt{A_{\mathrm{D}} / K_{\mathrm{D}}}$. If $t_{\mathrm{D}}$ is made thin, $\delta_{\mathrm{D}}$ at the surface becomes narrow, and the attraction decreases. Moreover, narrow $\delta_{\mathrm{D}}$ contributes to the increase in $M_{\mathrm{D}} H_{\mathrm{D}}$. This brings short $d_{\min }$. On the other hand, making $K_{\mathrm{D}}$ double, $\partial E_{\text {tot }} / \partial d$ became strong as shown in Fig. 8(c). Though $\delta_{\mathrm{D}}$ becomes thin by increasing $K_{\mathrm{D}}$ according to the relationship of $\delta_{\mathrm{D}} \approx \pi \sqrt{A_{\mathrm{D}} / K_{\mathrm{D}}}, \partial E_{\text {tot }} / \partial d$ increases with increasing $K_{\mathrm{D}}$ since $E_{\mathrm{tot}}$ increases itself. Concerning the stability of the domain, the increase of $M_{\mathrm{D}} H_{\mathrm{D}}$ is much more than that of $\partial E_{\text {tot }} / \partial d$ due to the relationship of $M_{\mathrm{D}} H_{\mathrm{D}} \propto K_{\mathrm{D}}$ and $E_{\text {tot }} \propto \sqrt{K_{\mathrm{D}}}$. Of course narrow $\delta_{\mathrm{D}}$ contributes to the increase in $M_{\mathrm{D}} H_{\mathrm{D}}$. That also brings short $d_{\min }$.

In the above discussions, we did not take reverse time of the magnetic moment into consideration. If the noncoupling region can reach the left wall before the domain collapses, the domain wall displacement may be possible. So let us consider the time taken for the domain to collapse. In the case of $d=68 \mathrm{~nm}$, when the non-coupling region reaches the position of $35 \mathrm{~nm}$ from the left wall, the domain collapsed. Here, the energy difference $\Delta E_{\text {tot }}$ between the total energies of all cells before and after collapse of the domain, that is to say, the energy difference between the two domain walls and the interface wall was estimated as $1.58 \times 10^{-6} \mathrm{erg} / \mathrm{cm}$. If $\Delta E_{\text {tot }}$ is converted into magnetic field $H_{\mathrm{D}}$, it can be estimated as

$$
H_{\mathrm{D}}=\frac{\Delta E_{\mathrm{tot}}}{2 M_{\mathrm{D}} t_{\mathrm{D}} d}=968 \mathrm{Oe} \text {, }
$$

where $M_{\mathrm{D}}$ was set to $40 \mathrm{emu} / \mathrm{cm}^{3}$. Since $H_{\mathrm{D}}$ is considered to be higher than the Walker breakdown field, the time $\tau$ which is spent on rotation of the moment to the direction of magnetic field is given by ${ }^{6}$

$$
\tau=\frac{1+\alpha^{2}}{\alpha} \cdot \frac{1}{\eta / H_{\mathrm{D}}}
$$

where $\gamma$ and $\alpha$ are gyromagnetic ratio and damping factor, respectively. By substituting $\gamma=1.76 \times 10^{7} \mathrm{Oe}^{-1} \mathrm{sec}^{-1}$, 
Table 1 (a) The minimum mark length for which the domain wall displacement occurs in the front process. (b) The maximum mark length with which no ghost signal is reproduced in the rear process.

\begin{tabular}{|c|c|c|c|c|c|}
\hline \multirow{2}{*}{\multicolumn{2}{|c|}{$\mid \frac{(1)}{D(30 \mathrm{~nm})}$}} & \multirow{2}{*}{$\mid \frac{(2)}{D(20 \mathrm{~nm})}$} & \multirow{2}{*}{$\frac{(3)}{\mathrm{D}(30 \mathrm{~nm})}$} & \multirow{2}{*}{$\frac{(4)}{\mathrm{D}(30 \mathrm{~nm})}$} & \multirow{2}{*}{$\begin{array}{l}(5) \\
K_{\mathrm{D}} \rightarrow 2 K_{\mathrm{D}}\end{array}$} \\
\hline & & & & & \\
\hline & $2(10020$ & & 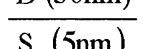 & $\mathrm{C}(5 \mathrm{~nm})$ & $\mathrm{D}(30 \mathrm{~nm})$ \\
\hline & (10iा & & $\frac{\mathrm{s} \text { (Jim }}{M}$ & $\mathrm{~S}(5 \mathrm{~nm})$ & $\mathrm{S}(10 \mathrm{~nm})$ \\
\hline & 101 & & & $\mathrm{M}$ & \\
\hline (a) & & & & $66 \mathrm{~nm}$ & $60 \mathrm{~nm}$ \\
\hline$(\mathrm{b}$ & $290 \mathrm{~nm}$ & $210 \mathrm{~nm}$ & $250 \mathrm{~nm}$ & $780 \mathrm{~nm}$ & $550 \mathrm{~nm}$ \\
\hline
\end{tabular}

$\alpha=10 / M_{\mathrm{D}}=0.25$, and $H_{\mathrm{D}}=968$ Oe for Eq. (4), $\tau$ is found as $0.249 \mathrm{nsec}$. Since the left wall start to displace when the non-coupling region reaches the position of 25 $\mathrm{nm}$ from the left wall, the time to move from $35 \mathrm{~nm}$ to 25 $\mathrm{nm}$ for the medium is estimated as $3.3 \mathrm{nsec}$. Since this time is much longer than $\tau$, it is considered that the calculation results are valid.

\subsection{The rear process}

Figure 9 shows the calculation results of wall structure in the rear process. The right side of Fig. 9 is high temperature region, and the temperature of region painted with hatch is the Curie temperature or higher. Layer structure in Fig. 9(a) is the same as Fig. 5. In the case of $d=250 \mathrm{~nm}$, when the left wall in the M-layer entered the coupling region, the interface wall was formed in the Slayer (1). When the non-coupling region reaches the position of $330 \mathrm{~nm}$ from the left wall, the interface wall (a)
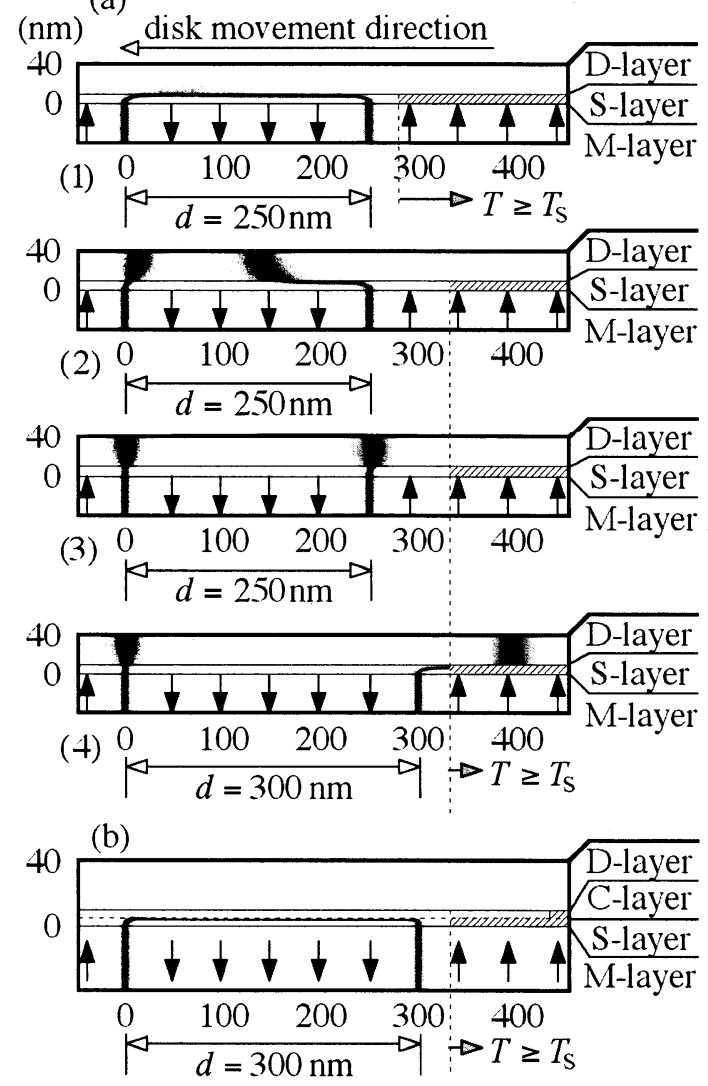

Fig.9 Wall structure on (a) the D/S/M structure and (b) the $\mathrm{D} / \mathrm{C} / \mathrm{S} / \mathrm{M}$ structure in the rear process. began to seep in the D-layer, and finally, two walls were created in the D-layer (2). In this case, the right wall in the D-layer was caught by the right wall in the M-layer (3). In the case of $d=300 \mathrm{~nm}$, however, the right wall which created in the D-layer displaced by the temperature gradient (4). That is the ghost phenomenon. Figure 9(b) shows the case of the $\mathrm{D} / \mathrm{C} / \mathrm{S} / \mathrm{M}$ structure on which the thicknesses of the D-layer, C-layer and S-layer are $30 \mathrm{~nm}, 5 \mathrm{~nm}$ and $5 \mathrm{~nm}$, respectively. Though the non-coupling region reaches the position of $330 \mathrm{~nm}$ from the left wall, the interface wall still stayed in the S-layer. The interface wall in the S-layer is disturbed to spread into the D-layer by the C-layer, since the wall energy density in the C-layer is higher than that in the S-layer as shown in Fig. 4(b). In this case, the ghost signal was suppressed up to the mark length of $800 \mathrm{~nm}$. The results mentioned above is almost the same as the experimental results reported ${ }^{1), 2)}$. The minimum mark length for which the domain wall displacement occurs in the front process, and the maximum mark length with which no ghost signal is reproduced in the rear process are summarized in Table 1. By making the D-layer (2) and the S-layer (3) thin, the maximum mark length in the rear process was shortened. In the case that the anisotropy energy constant in the D-layer was doubled intentionally (5), both the critical mark lengths in the front process and rear process are improved than (1).

\section{Conclusions}

We simulated wall structure and energy on the DWDD, and considered the front process and the rear process under the temperature gradient. For the front process, when the mark length was comparable with the domain wall width in the D-layer, the domain copied on the D-layer collapses. By making the D-layer thin, the minimum mark length for which the domain wall displacement occurs can be shortened. For the rear process, by inserting the C-layer between the D-layer and the S-layer, the mark length with which the ghost signal was suppressed became longer. By making the anisotropy energy constant in the D-layer increase, improvements of the front process and rear process on the DWDD will be expected.

\section{References}

1) T. Shiratori, E. Fujii, Y. Miyaoka, and Y. Hozumi: Proc. Magneto-Optical Recording Int. Symp., Yamagata, 1997, J. Magn. Soc. Jpn., 22 (Suppl. No. S2), 47 (1998).

2) T. Shiratori, E. Fujii, Y. Miyaoka, Y. Hozumi, T. Kobayashi, and M. Masuda: Dig. Magneto-Optical Recording Int. Symp., Monterey, 1999, p. 60 (Magnetics Society of Japan).

3) Y. Nakatani, T. Kobayashi, and T. Shiratori: 24 th Conference on Magnetics Japan, Tokyo, 2000, p. 443 (Magnetics Society of Japan, Tokyo, 2000).

4) T. Kobayashi, M. Masuda, and T. Shiratori: J. Magn. Soc. Jpn., 25, 371 (2001).

5) M. Mansuripur, and M. Ruane: IEEE Trans. Magn., MAG-22, 33 (1986).

6) For example, S. Uchiyama and M. Masuda: Magnetic Materials, p. 127 (Corona Publishing, Tokyo, 1980)

Received April 28, 2004; Accepted July 26, 2004. 